\title{
UPLINK BEAMFORMING FOR THE FDD MODE OF UTRA
}

\author{
Xavier Mestre, Javier R. Fonollosa, Gregori Vázquez \\ Department of Signal Theory and Communications \\ Universitat Politècnica de Catalunya \\ c/Jordi Girona, 1-3, 08034 Barcelona, SPAIN \\ Phone: +34 934017052 Fax: +34 934016447 \\ e-mail: $\{$ mestre, fono, gregori\}@gps.tsc.upc.es
}

\begin{abstract}
This paper presents some link level simulation results for the evaluation of adaptive antennas in the uplink of the FDD mode of UTRA. Two families of algorithms were initially considered, the basic difference between them being their ability/disability to suppress the contribution from WCDMA directional interfering sources. Two distinct schemes were established as representatives for each family and their performance was evaluated in presence of some illustrative interfering scenarios. In the light of the results it is shown that time-reference beamforming algorithms suffer from severe beampattern distortion effects when applied as such. This in turn causes harsh performance degradation in terms of raw BER, especially at high SINR levels. It is shown that these shortcomings are essentially caused by the uplink multiplexation of the traffic channel, which is seen by the base station as a powerful interfering source coming from the direction of arrival of the desired user.
\end{abstract}

\section{INTRODUCTION}

The first aim of the work presented herein was to evaluate the convenience of array-processing interference canceling schemes in typical W-CDMA scenarios. Clearly, the performance of such architectures will depend strongly on both spatial distribution and temporal structure of the transmitted signals. Since the analytical modeling of these effects becomes somewhat burdensome, a first approximation to the problem by means of extensive simulations seemed most appropriate.

The evaluation process was carried out following FRAMES recommendations as described in [2]. Thus the "Actual Value Interface" (AVI) was chosen as the most suitable connection between link-level and system-level simulations. The technique establishes that the link level simulation results should be measured in a burst-by-burst basis so that the system simulator undertakes all coding and link level adaptation. Thanks to that, all radio resource management algorithms (having an activation period higher than burst duration) can be accurately simulated on the system level platform.

\section{CHANNEL AND SIGNAL MODEL}

We denote $\mathbf{x}(t)$ ( $P x \mathbf{l}$ column vector) the received snapshot at a particular time instant $t$. If $a_{\mathrm{s}}(t)$ represents the transmitted

This work was partially supported by the European Commission under ACTS Project AC347 SUNBEAM, the Spanish Government (TIC96-0500-C10-01, TIC98-0412, TIC98-0703) and the Catalan Government CIRIT 1998SGR-00081. (analytic) signal coming from the $s$-th mobile station, its contribution to the received snapshot can be modeled as follows:

$$
\mathbf{x}_{s}(t)=\sum_{n} g_{n} \mathbf{s}_{n}(t) a_{n}\left(t-\tau_{n}\right)
$$

being $g_{n}$ a complex propagation gain and $s_{n}(t)$ a time-varying superposition of steering vectors. This generalized steering vector $\mathbf{s}_{n}(t)$-as it is commonly referred to in the literatureincorporates the angular information of the channel and depends on time due to the Doppler effect.

In the sequel we will assume that 1 ) the received signal is properly sampled at a multiple of the chip rate (Nsc samples/chip) and 2) perfect synchronization with the user of interest has been attained already. Now, gathering $M$ consecutive snapshots of the received signal at the base station into an $M x P$ matrix $\mathbf{X}$, we have:

$$
\mathbf{X}=\left[\begin{array}{llll}
\mathbf{x}(0) & \mathbf{x}(T s) & \cdots & \mathbf{x}((M-1) T s)
\end{array}\right]^{T}
$$

with $T s$ denoting the sampling period. Let us now introduce an $M x L$ segment of convolution matrix:

$$
\mathbf{D}=\left[\begin{array}{cccc}
d((L-1) N s c+1) & d((L-2) N s c+1) & \cdots & d(1) \\
d((L-1) N s c+2) & d((L-2) N s c+2) & & d(2) \\
d((L-1) N s c+3) & & & d(3) \\
\vdots & \vdots & & \vdots \\
d((L-1) N s c+M) & d((L-2) N s c+M) & \cdots & d(M)
\end{array}\right]
$$

generated from $M+(L-1) N s c$ samples of the desired user's training sequence $d(t)$ transmitted in the Control Channel (PDCCH) -or a repetition thereof whenever $M+(L-1) N s c$ is bigger than the period of the training sequence-. Upon the definition of an $L x P$ matrix including the two-dimensional discrete (1 sample/chip) channel impulse response:

$$
\mathbf{H}=\left[g_{1} \mathbf{s}_{1} g_{2} \mathbf{s}_{2} \cdots g_{L} \mathbf{s}_{t}\right]^{T}
$$

we can express the spatial-temporal received signal matrix as:

$$
\mathbf{X}=\mathbf{D H}+\mathbf{W}
$$

where $\mathbf{W}(M x P)$ includes not only spatial-temporal background noise but also the contribution of signals transmitted from other mobile stations as well as traffic channels embedded in the desired user's data stream. Provided that the length of the channel assumed at the receiver $(L)$ is not sufficiently large to cope with the whole delay spread of the radio channel, the latest reflections of the incoming signal are assumed to be included in this noise term. 


\section{UPLINK ALGORITHMS UNDER TEST}

The main purpose of the considered simulations was to investigate the influence of different representative W-CDMA interfering scenarios on the performance of conventional adaptive antenna systems. The objective was to quantify the potential advantages of interference canceling schemes in front of purely diversity combining architectures. Thus, two different families of algorithms were considered, each one resorting to a distinct presumption concerning the spatial nature of interference.

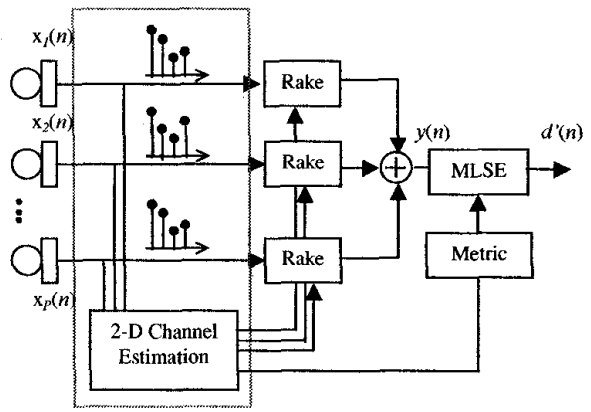

Figure 1. Vectorial Rake Receiver

The first scheme under consideration, hereinafter referred to as $V$-Rake Receiver (Figure 1), was chosen as representative for the family of algorithms which do not take into account the presence of directional interfering sources. The scheme can be basically shown to be the optimum detector from a maximumlikelihood point of view when only omnidirectional Gaussian noise is present in the scenario. Assuming that the components of the matrix $\mathbf{W}$ are jointly Gaussian-distributed, the receptor can be derived from the maximization of the following likelihood function:

$$
\eta=\operatorname{vec}^{H}(\mathbf{X}-\mathbf{D H}) \mathbf{R}_{w}^{-1} v e c(\mathbf{X}-\mathbf{D H})
$$

with $\mathbf{R}_{w}$ the MPxMP covariance matrix of $v e c(\mathbf{W})$, and $\otimes$ and $v e c()$ denoting the Kronecker product and the column-wise matrix stacking operation respectively. After some manipulation of (6) we get:

$$
\begin{gathered}
\eta=v e c^{H}(\mathbf{X}) \mathbf{R}_{w}^{-1} \operatorname{vec}(\mathbf{X})+2 \operatorname{Re}[y]+ \\
+\operatorname{vec}^{H}(\mathbf{D}) \mathbf{R} v e c(\mathbf{D})
\end{gathered}
$$

where we have defined:

$$
\begin{gathered}
y=\operatorname{vec}^{H}(\mathbf{D H}) \mathbf{R}_{w}^{-1} \operatorname{vec}(\mathbf{X}) \\
\mathbf{R}=\left(\mathbf{H}^{*} \otimes \mathbf{I}_{M}\right) \mathbf{R}_{w}^{-1}\left(\mathbf{H}^{T} \otimes \mathbf{I}_{M}\right)
\end{gathered}
$$

with $\mathbf{I}_{M}$ the $M x M$ identity matrix. This formulation allows separation of the combining part of the receiver from its subsequent data-detection part. Provided that noise and interference can be considered spatially and temporally uncorrelated, $y$ becomes equal to (up to a scalar factor):

$$
y=\operatorname{vec}^{H}(\mathbf{D H}) \operatorname{vec}(\mathbf{X})=\operatorname{tr}\left[(\mathbf{D H})^{H} \mathbf{X}\right]
$$

If we consider the following decomposition of the channel and input signal matrices:

$$
\begin{aligned}
& \mathbf{H}=\left[\begin{array}{lll}
\mathbf{h}_{1} & \cdots & \mathbf{h}_{p}
\end{array}\right] \\
& \mathbf{X}=\left[\begin{array}{lll}
\mathbf{x}_{1} & \cdots & \mathbf{x}_{p}
\end{array}\right]
\end{aligned}
$$

the scalar $y$ can be expressed in the following way:

$$
y=\sum_{p=1}^{P} \mathbf{h}_{p}^{H} \mathbf{D}^{H} \mathbf{x}_{p}
$$

Each term $\mathbf{h}_{p}^{H} \mathbf{D}^{H} \mathbf{x}_{p}$ can be interpreted as the output of a Rake receiver matched to each antenna channel impulse response. In conclusion, the optimum receiver results in the scheme herein regarded as the $V$-Rake Receiver (see Figure 1). Yet one issue remains open: since the channel impulse response $\mathbf{H}$ is not known, it must be estimated from the incoming data. An ML channel estimate can be obtained taking derivatives of the cost function (6) with respect to $\operatorname{vec}\left(\mathbf{H}^{*}\right)$ and forcing the corresponding gradient to be zero:

$$
\begin{gathered}
\nabla_{v e c\left(\mathbf{H}^{\circ}\right)} \eta=\left(\mathbf{I}_{L} \otimes \mathbf{D}^{H}\right) \mathbf{R}_{w}^{-1}\left(\mathbf{I}_{L} \otimes \mathbf{D}\right) \operatorname{vec}(\mathbf{H}) \\
-\left(\mathbf{I}_{L} \otimes \mathbf{D}^{H}\right) \mathbf{R}_{w}^{-1} \operatorname{vec}(\mathbf{X})=\mathbf{0}
\end{gathered}
$$

from where we obtain:

$$
\operatorname{vec}(\mathbf{H})=\left[\left(\mathbf{I}_{L} \otimes \mathbf{D}^{H}\right) \mathbf{R}_{w}^{-1}\left(\mathbf{I}_{L} \otimes \mathbf{D}\right)\right]^{-1}\left(\mathbf{I}_{L} \otimes \mathbf{D}^{H}\right) \mathbf{R}_{w}^{-1} \operatorname{vec}(\mathbf{X})(13)
$$

Finally, for the $V$-Rake Receiver considered here:

$$
\mathbf{H}=\left(\mathbf{D}^{H} \mathbf{D}\right)^{-1} \mathbf{D}^{H} \mathbf{X}
$$

The approximation of temporal uncorrelation of interfering components of the input signal seems reasonable as long as we deal with digitally modulated signals sampled at the chip rate. On the contrary, their spatial whiteness can not be so easily justified and will depend strongly on the actual scenario under consideration. A more sensible approximation, judging by conclusions drawn from measurement campaigns in [7], is to consider that the structure of the distinct generalized steering vectors $s_{l}$ in (4) does not vary in observation intervals shorter than the delay spread of the mobile radio channel. This is equivalent to the approximation:

$$
\begin{gathered}
\mathbf{H} \approx \mathbf{h}_{0} \mathbf{s}_{0}^{T} \\
\operatorname{vec}(\mathbf{H}) \approx \mathbf{s}_{0} \otimes \mathbf{h}_{0}
\end{gathered}
$$

where $\mathbf{h}_{0}$ and $\mathbf{s}_{0}$ represent the equivalent one-dimensional channel impulse response and generalized steering vector respectively. Substitution of (15) into (8) leads to:

$$
y=\left(\mathbf{s}_{0} \otimes \mathbf{h}_{0}\right)^{H}\left(\mathbf{I}_{P} \otimes \mathbf{D}^{H}\right) \mathbf{R}_{w}^{-1} \operatorname{vec}(\mathbf{X})
$$

or, after some algebra:

$$
y=\left(\mathbf{R}_{w t}^{-1} \mathbf{D} \mathbf{h}_{0}\right)^{H} \mathbf{X}\left(\mathbf{R}_{w s}^{-1} \mathbf{s}_{0}\right)^{*}
$$

where, by virtue of the spatial-temporal separateness approximation, we have decomposed the non-desired signal covariance matrix into its spatial and temporal components $\mathbf{R}_{w}=\mathbf{R}_{w s} \otimes \mathbf{R}_{w t}$. We observe that the optimum combining prior to the sequence detection collapses into two separate stages: a spatial stage, in which the incoming snapshots are preprocessed by the optimum beamforming (in the sense that it provides maximum Signal to Interference plus Noise Ratio SINR at its 
output); and a temporal stage, which can be interpreted as a single Rake receiver.

In this context, the second algorithm under test the so-called Matched Desired Impulse Response MDIR Receiver [8][3] (Figure 2)- resorts to the separateness approximation in (15) in order to provide the ML estimation of the transmitted data, the beamformer weights and the equivalent channel $\mathbf{h}_{0}$. Assuming Gaussianity of the non-desired interferences the log-likelihood function of the received data after the spatial processing can be expressed (up to a constant) as:

$$
\zeta=(\mathbf{X b}-\mathbf{D h})^{H} \mathbf{R}_{w \mathrm{~s}}^{-1}(\mathbf{X b}-\mathbf{D h})
$$

where we have considered that the channel impulse response after the beamforming process $\mathbf{h}$, the data, and the beamformer itself $\mathbf{b}$ are all deterministic quantities. As already discussed, it seems reasonable to assume the spatial covariance matrix is close to the identity so that the ML cost function turns out to be a mean square error measure:

$$
\zeta=\|\mathbf{X b}-\mathbf{D h}\|^{2}
$$

In order to avoid the trivial solution an additional constraint must be imposed. For instance, one may set the power associated with the training sequence at the output of the beamformer equal to constant:

$$
\mathbf{b X}^{H} \mathbf{D}\left(\mathbf{D}^{H} \mathbf{D}\right) \mathbf{D}^{H} \mathbf{X b}=\mathbf{1}
$$

Thereby, using the Lagrange multipliers method the solution may be shown to be [3]:

$$
\begin{aligned}
\mathbf{X}^{H} \mathbf{X b} & =\lambda_{\min } \mathbf{X}^{H} \mathbf{D}\left(\mathbf{D}^{H} \mathbf{D}\right)^{-1} \mathbf{D}^{H} \mathbf{X b} \\
\mathbf{h} & =\left(\mathbf{D}^{H} \mathbf{D}\right)^{-1} \mathbf{D}^{H} \mathbf{X b}
\end{aligned}
$$

Thus, the beamforming weight vector is found as the eigenvector corresponding to the minimum generalized eigenvalue of the matrix pencil $\left\{\mathbf{X}^{H} \mathbf{X}, \mathbf{X}^{H} \mathbf{D}\left(\mathbf{D}^{H} \mathbf{D}\right)^{-1} \mathbf{D}^{H} \mathbf{X}\right\}$. Since this eigenvalue is equal to the inverse of the output SINR minus one, the solution is yielding optimality in terms of SINR before the Rake receiver.

\section{SIMULATION ASPECTS}

The main difficulty that arises when simulating a W-CDMA environment resides in taking into account all possible bit rates, and consequently spread factors, that might be involved in an actual scenario. In order to overcome this problem two different kinds of users were defined, the difference between them being basically the type of service that they require: High Bit Rate (LBR) and Low Bit Rate (HBR) users. The former are supposed to have a higher bandwidth demand and, consequently, an actual mobile network will be capable of handling a high number of LBR users whereas the number of mobiles requiring a HBR service will be limited. Furthermore, the higher the bit rate, the more transmitted power is needed in order to preserve a given quality of service. All this suggests that LBR users can be properly modeled as omnidirectional Gaussian noise. In our case, a constant spread factor (SF) was assigned to all HBR users irrespectively of whether they constitute desired or interfering sources.
Concerning the signal generation in the simulations, each sensor frequency-selective channel was modeled with a tapped delay line of time-varying coefficients as shown in (1). Particularly, the models referred to as Outdoor to Indoor and Pedestrian and Vehicular in [6] were considered for the generation of the channel frequency selectivity, whereas the angular approach presented in [7] was used to characterize the angular dispersion of the propagation process. Thus each flatfading component of the mobile channel was generated using a ray model, where the number of impinging waveforms was set equal to a Poisson random variable (mean equal to 25 rays). In addition, a Laplacian Power Angular Spectrum was considered, along with a Gaussian distribution of the different directions of arrival for each user. The Power Angular Spread was fixed to 8 degrees for all taps and scenarios. The mobile speed was set to $3 \mathrm{~km} / \mathrm{h}$ and $120 \mathrm{~km} / \mathrm{h}$ for the Pedestrian and Vehicular models respectively. According to the Actual Value Interface, the simulation duration was set equal to the minimum power control actualization period, namely $0.625 \mathrm{~ms}$.

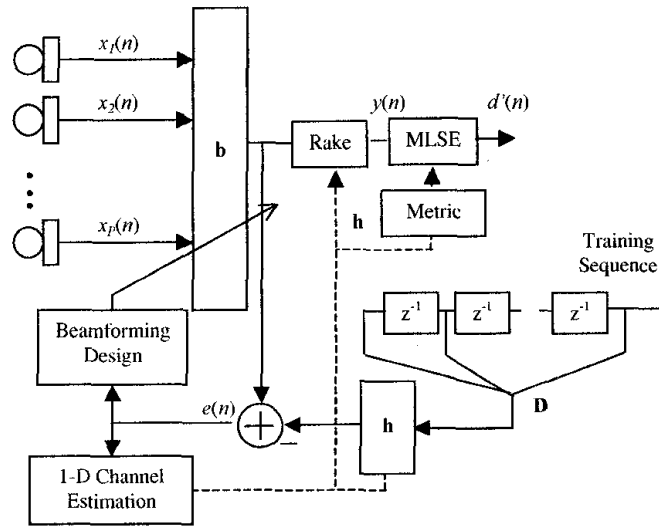

Figure 2. Matched Desired Impulse Response Receiver.

All the array-processing algorithms were simulated on a linear equally spaced array of 8 antennas, in which the interelement separation was set to half wavelength at the carrier frequency $(1950 \mathrm{MHz})$. All mobile angular locations were arbitrarily set by a uniform random variable within $[-60,60]$ degrees, i.e. 120 degrees sectorization was assumed. Each user was supposed to generate a single Dedicated Physical Data Channel (DPDCH) together with its associated Dedicated Physical Control Channel DPCCH (see [9] for details of the modulation formats).

\section{SIMULATION RESULTS}

Two interfering scenarios were simulated for each power delay profile model, one with a single dominating HBR interference and another with five. Apart from the array beamforming algorithms presented in section 3, a single-sensor Rake receiver was considered for comparison purposes.

Uplink results are depicted in terms of raw (uncoded) BER in Figure 3 and Figure 4. Although simulations took into account a high range of LBR power (reflected in the $\mathrm{Eb} / \mathrm{No}$ ratio), only results with $\mathrm{Eb} / \mathrm{No}=10 \mathrm{~dB}$ are presented herein. Note that the $\mathrm{BER}$ is always expressed as a function of the instantaneous $\mathrm{Eb} / \mathrm{Io}$ per sensor (i.e., received by a single antenna and 
measured within an actualization period of the fast power control). Thanks to that, a conventional planning tool can directly use these results and therefore disregard the existence of adaptive antennas.

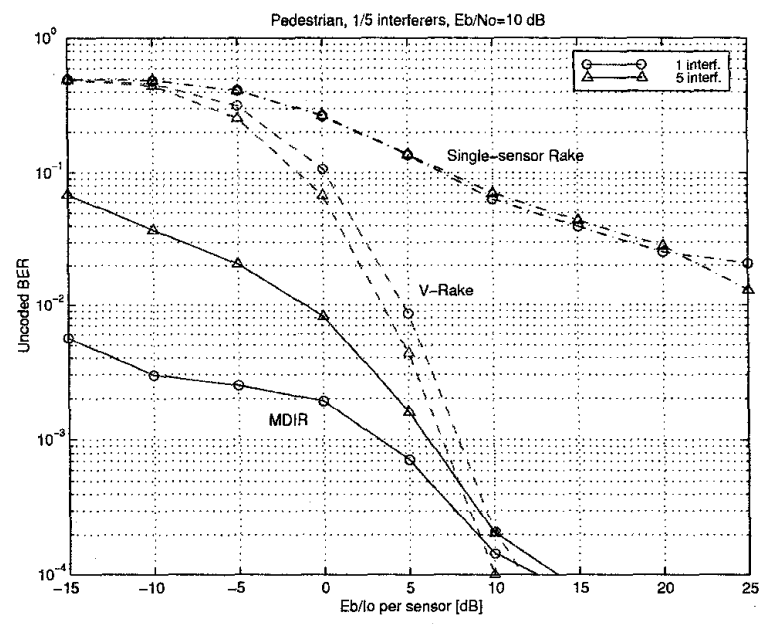

Figure 3. Uplink results for the Pedestrian Channel Model.

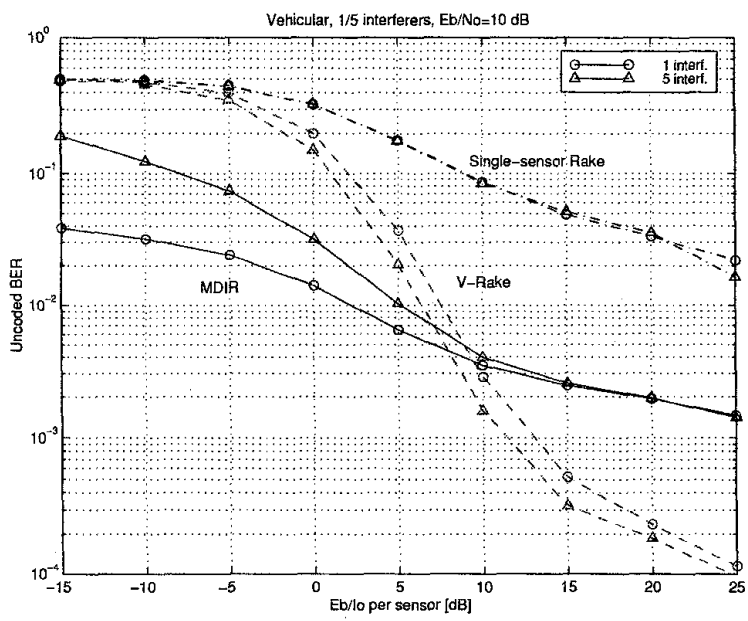

Figure 4. Uplink results for the Vehicular Channel Model.

Comparing the performance of the proposed algorithms, the MDIR receiver generally attains the best results in terms of required $\mathrm{Eb} / \mathrm{No}$ and $\mathrm{Eb} / \mathrm{Io}$ for a particular raw $\mathrm{BER}$ in the range of interest (usually from $10^{-2}$ to $10^{-1}$ for raw BER). This is a logical result, since neither the V-Rake algorithm nor its singlesensor counterpart take into consideration the presence of interference, which has a most detrimental effect on the receiver performance. Note however that for high $\mathrm{Eb} / \mathrm{No}$ or $\mathrm{Eb} / \mathrm{Io}$ levels the Multisensor Rake receiver actually outperforms the MDIR scheme, the reason for that being basically twofold. First, the MDIR strategy bases its operation upon the adaptation of a narrowband beamformer, an approximate solution to the impairments introduced by the time-dispersive channel. Second, non-ideal estimates of the correlation matrices are bound to originate significant performance losses, especially when the power of the received signals is high enough.

The loss introduced by the approximation by the use of a narrowband beamformer instead of an array of FIR filters (approach equivalent to that of a wideband beamformer) becomes fully justified by the front-end complexity reduction that the former entails. Of more serious concern is the degradation introduced by imperfect estimation of the correlation matrices needed in the MDIR algorithm. Basically, the reason for this behavior is that the traffic channel multiplexed with the pilot is seen by the base station as an interfering signal coming from the very same angular direction (note that only the training sequence component is identified as 'desired source' by the beamforming algorithm). This leads to a degradation of the desired signal estimated covariance matrix equivalent to a random pointing error when tackling with conventional sources. Besides, the higher the desired received power (and so the measured $\mathrm{Eb} /(\mathrm{No}+\mathrm{Io})$ ) the more precise the estimation has to be in order to prevent the desired signal from being cancelled out by the beamformer [1]. Since accuracy is in this case limited by the interference of the traffic channel, at high $\mathrm{Eb} /(\mathrm{No}+\mathrm{Io})$ ratios the system can not avoid suppressing the desired signal instead of enhancing it.

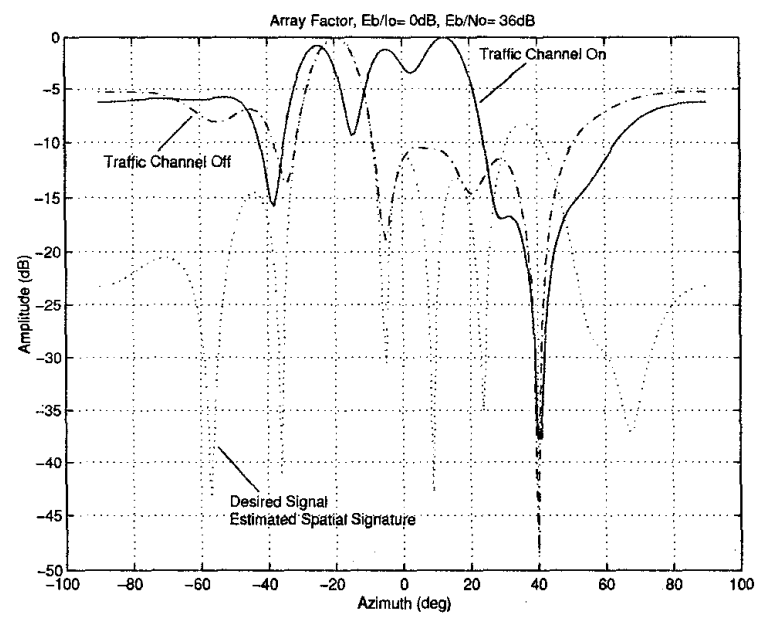

Figure 5. Degradation of the array response due to the presence of the traffic channel. Solid and dash-dotted line: spatial response of the global beamformer. Dotted line: spatial response of the eigenvector corresponding to the maximum eigenvalue of the desired signal covariance matrix. Desired signal DOA: -20 deg. Interfering signal DOA: 40 deg.

The consequences of this effect on the array spatial response can be noticed in Figure 5, where the array factor has been represented for a Pedestrian scenario in presence of a high level of desired signal power. In order to obtain more insight into the beampattern distortion effects, a low angular spread was chosen (0.01 degrees). It may initially seem that when the training sequence is transmitted together with a traffic channel the scheme can still estimate the desired signal spatial signature (dotted line) with a certain degree of accuracy. However the global beamformer spatial responses show that small errors in this estimate result in great distortion effects of the global beampattern, what in turn brings about high degradation of the BER at the mobile station. 
Several possible solutions to this distortion effect are currently being under investigation. They are based on semi-blind estimation techniques and basically seek to take into account not only the training sequence but also other unknown interfering symbols embedded in the modulated data stream [4]. The formulation of these algorithms is not reported here for lack of space, although we do include preliminary results to demonstrate their potential. In particular, Figure 6 shows the output SINR of the beamformer as a function of the input power level (with respect to the background noise) in an scenario with two $\mathrm{HBR}$ users transmitting with $\mathrm{SF}=8$. As the power of the HBR users increases beyond $10 \mathrm{~dB}$, proper output SINR values can no longer be sustained. Nevertheless, this proclivity is successfully overcome by means of the application of a semiblind technique based on a Conditioned ML criterion. The interested reader is referred to [4] for further details of the algorithm as well as more extensive performance simulation results.

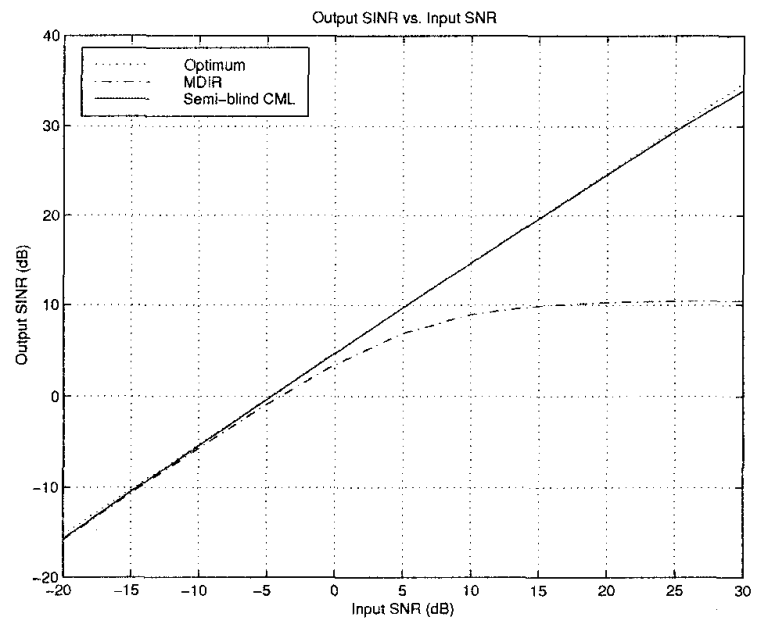

Figure 6. Output SINR versus Input SNR in a scenario with two $\mathrm{HBR}$ users transmitting with $\mathrm{SF}=8$ and the same power.

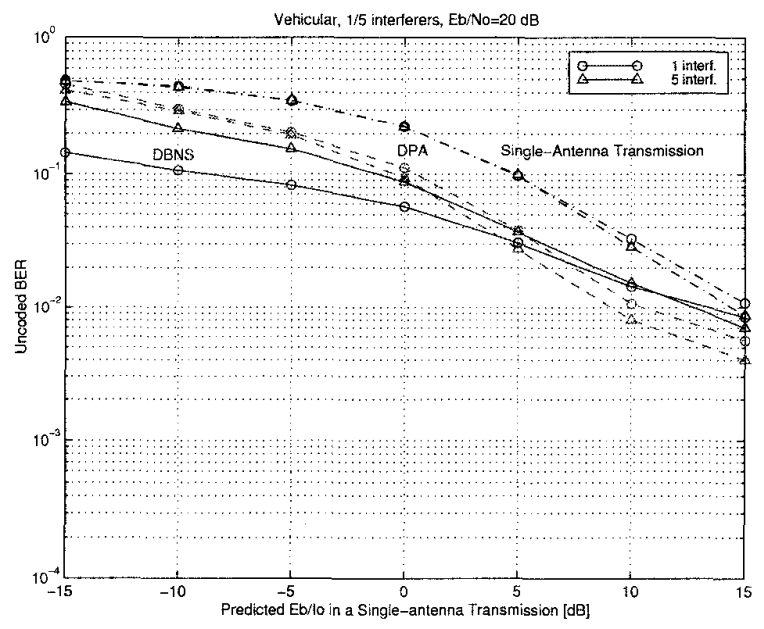

Figure 7. Downlink results for the Vehicular Model.

In addition to the work presented here, link-level simulations of downlink array-processing algorithms for the FDD mode of UTRA have also been performed (see [5] for details). Figure 7 presents performance curves for two different beamforming algorithms: a pointing scheme (DPA) and a null-steering scheme (DBNS). The DPA approach seeks to generate a maximum array response towards the direction of arrival of the desired user whereas the DBNS deployment is particularly intended to maximize the SINR at the mobile station input. Surprisingly, it can be observed that the DPA algorithm outperforms the DBNS scheme for values of the instantaneous $\mathrm{Eb} / \mathrm{Io}$ above 5-8 $\mathrm{dB}$. Once again, this behavior is basically motivated by a poor estimation of the desired signal spatial signature. As already shown, this estimation is precise enough to properly point at the desired user, but not so reliable to avoid the desired signal suppression when a maximum SINR strategy is adopted.

\section{CONCLUSIONS}

This paper has presented some link-level simulation results for the uplink and downlink of the current UTRA-FDD standard definition. Results show that application of classical timereference beamforming techniques encounters severe performance problems when operating at high input SNR levels. A beampattern distortion effect, principally induced by the multiplexed traffic channel, has been shown to be the ultimate responsible for the performance degradation. Finally, semi-blind techniques are put forward as valid architectures to take into account the presence of the auto-interfering channel. Performance results of these techniques will be further addressed in [4].

\section{REFERENCES}

[1] Compton R. T., "Pointing Accuracy and Dynamic Range in a steered beam adaptive array", IEEE Trans. on AES, vol. AES-16, 1976, pp. 280-287.

[2] Hämäläinen S., Slanina P., Hartman M., Lappeteläinen A., Holma H., Salonaho O., "A Novel Interface between Link and System Level Simulations", Proc. ACTS Mobile Telecomm. Summit, Aalborg, Denmark, pp. 599-604, October 1997.

[3] Lagunas M.A., Pérez A. and Vidal J., "Joint Beamforming and Viterbi Equalization in Wireless Communications", Proc. $31^{\text {st }}$ Asilomar Conference on Signals, Systems and Computers, Pacific Grave, California, November 1997.

[4] Mestre X., Fonollosa J. R., Antón C., "Adaptive Beamforming for HBR services in the FDD mode of UTRA", submitted to the IEEE Vehicular Technology Conference, 1999-Fall, Amsterdam, The Netherlands.

[5] Mestre X., Fonollosa J.R, "Performance Evaluation for UTRAFDD", Proc. FRAMES Workshop, Delft (The Netherlands), January 1999.

[6] Nikula E., "Air Interface Specification, Layer 1, Draft", Intermediate Deliverable AC090/NOK/PT0/DS/R/014/bl. FRAMES project.

[7] Pedersen K.I., Mogensen P.E., Fleury B.H., "A Stochastic Model of the Temporal and Azimuthal Dispersion seen at the Base Station in Outdoor Propagation Environments", submitted to the IEEE Trans. on Vehicular Technology.

[8] Pipon E.P., Chevalier P., Villa P., Monot J.J, “Joint Spatial and Temporal Equalization for channels with ISI and CCI- Theoretical and Experimental results for a base station reception", Proc. IEEE Signal Processing Workshop on Signal Processing Advances in Wireless Communications, Paris, France, April 1997, pp.309-312.

[9] "Submission of Proposed Radio Transmission Technologies: the ETSI UMTS Terrestrial Radio Access (UTRA) ITU-R RTT Candidate Submission", ETSI SMG2. Date of submission: 29/1/1998. Available at the ITU WWW http://www.itu.ch/imt/ 